\title{
New Removal Operators for Surface Skeletonization
}

\author{
Carlo Arcelli, Gabriella Sanniti di Baja, and Luca Serino \\ Institute of Cybernetics "E.Caianiello", CNR, Pozzuoli (Naples), Italy \\ (c.arcelli, g.sannitidibaja, 1.serino) @cib.na.cnr.it
}

\begin{abstract}
New $3 \times 3 \times 3$ operators are introduced to compute the surface skeleton of a 3D object by either sequential or parallel voxel removal. We show that the operators can be employed without creating disconnections, cavities, tunnels and vanishing of object components. A final thinning process, aimed at obtaining a unit-thick surface skeleton, is also described.
\end{abstract}

\section{Introduction}

Topology preserving removal operations have received much attention in the literature dealing with discrete objects, since their use is crucial for processes such as shrinking, thinning and skeletonization. In this respect, the interest is towards operations based on a small neighborhood of an object element $p$ (the $3 \times 3$ neighborhood in $2 \mathrm{D}$, and the $3 \times 3 \times 3$ neighborhood in $3 \mathrm{D}$ ). In fact, it has been proved that if removal of $\mathrm{p}$ does not alter the topology in the neighborhood of $\mathrm{p}$, then $\mathrm{p}$ can be safely removed since topology is also globally maintained $[1,2]$.

To preserve topology in 2D, removal of $\mathrm{p}$ from an 8-connected object should not create holes or disconnections. In other words, $\mathrm{p}$ should be a simple point. Removal of $\mathrm{p}$ does not create holes, if $\mathrm{p}$ has at least a 4-adjacent neighbor in the background. Disconnections do not occur if $\mathrm{p}$ has exactly one 8-connected component of object neighbors. In 3D, topology preserving removal operations can still be based on the notion of simple point $[3,4]$, but besides cavities and disconnections, also tunnels have to be taken into account in the definition of point simplicity. Similarly to the 2D case, cavities are not created by removing $\mathrm{p}$ from a 26 -connected object if $\mathrm{p}$ has at least a 6adjacent neighbor (face-neighbor) in the background. To this purpose, the number of 26-connected components of object voxels and the number of 6-connected components of background voxels have to be computed by processing the $3 \times 3 \times 3$ neighborhood of $p$. These numbers can be used to determine whether removal of $p$ causes disconnections. Moreover, removal of $\mathrm{p}$ should prevent creation of tunnels. In other words, removal of $\mathrm{p}$ from a tunnel-free solid object should not change the object into one that cannot be deformed to a single voxel [5]. To this aim, the number of 6connected components of background voxels, having $\mathrm{p}$ as face-neighbor and computed in the $3 \times 3 \times 3$ neighborhood of $p$ deprived of the eight vertex-neighbors, has to be computed.

In this paper, we introduce a set of $3 \times 3 \times 3$ topology preserving removal operations, based on the configurations of the voxels in the neighborhood of $\mathrm{p}$. We show that 
these operations can be used in the framework of 3D surface skeletonization. In particular, we introduce two surface skeletonization algorithms using respectively sequential and parallel voxel removal.

\section{Notions and Definitions}

We work with a binary finite voxel image in a cubic grid, where the object is the set of 1's and the background is the set of 0's. As implicitly stated in the previous section, we choose the 26-connectedness for the object and the 6-connectedness for the background. The object consists of border voxels, i.e., those having a face-neighbor in the background, and inside voxels, i.e., those having no face-neighbors in the background. Any voxel $p$ has 6 face-, 12 edge- and 8 vertex-neighbors. We call $\mathrm{N}(\mathrm{p})$ the $3 \times 3 \times 3$ set including $\mathrm{p}$ and its 26 neighbors, and $\mathrm{N}(\mathrm{p}) *$ the set $\mathrm{N}(\mathrm{p})$ deprived of the vertex-neighbors.

A 6-connected path of voxels is termed face-aligned if no change of direction is allowed along the path. Three directions, each with two orientations, are possible.

We say that a transition exists for a border voxel $\mathrm{p}$, if a face-aligned path consisting of three voxels can be found, where $p$ is the intermediate voxel and the two extremes of the path are respectively an inside voxel and a background voxel.

A protrusion is a maximal connected set of border voxels, none of which has an inside voxel as face-neighbor. By this definition it follows that a protrusion is the at most 2-voxel thick union of surface-like and curve-like sets.

A cavity is a 6-connected component of the background fully surrounded by the object [6].

A tunnel exists if a path can be found in the object that cannot be deformed to a single voxel [5].

An object voxel $\mathrm{p}$ is simple if the object including $\mathrm{p}$ is homotopic to the object deprived of $p$. Simplicity of $p$ means that the numbers of cavities, object components, and tunnels are the same, independently of whether $p$ is in the object or in the background. Four main conditions are satisfied in $\mathrm{N}(\mathrm{p})$ by a simple voxel $\mathrm{p}: 1) \mathrm{p}$ is not an inside voxel (cavity prevention condition); 2) the number of 26-connected object components in $\mathrm{N}(\mathrm{p})$ is equal to 1 (object connectedness condition); 3 ) the number of 6-connected components of background voxels in $\mathrm{N}(\mathrm{p})$ is equal to 1 (background connectedness condition); and 4) the number of 6-connected components of background voxels, having $\mathrm{p}$ as a face-neighbor and computed in $\mathrm{N}(\mathrm{p})^{*}$ is equal to 1 (tunnel prevention condition).

We point out that voxel configurations, in correspondence of which tunnels risk to be created in the object by removal of $\mathrm{p}$, may exist when in $\mathrm{N}(\mathrm{p})^{*}$ two background face-neighbors of $\mathrm{p}$ belonging to distinct 6-connected components either form a facealigned path with $\mathrm{p}$ (type 1), or are edge-neighbors of each other (type 2).

For completeness, we note that a voxel $\mathrm{p}$ whose 26 neighbors are all background voxels, i.e., an isolated voxel, is not simple.

The distance transform of an image is a labeled version of the image where the label of any voxel is the distance of that voxel from the background. In this paper we compute the distance transform DT by using the distance $\mathrm{D}^{6}$, which is the $3 \mathrm{D}$ version of the well-known city-block distance in 2D. For the computation of DT, as well as of 
distance transforms based on other metrics, see [7]. Voxels in DT can be interpreted as centers of balls with radius equal to the distance label.

A center of a maximal ball, CMB, is an object voxel whose associated ball is not included by any other single ball in the object. In DT, a CMB is any voxel whose distance label is not smaller than the labels of its 6 face-neighbors. The union of all the maximal balls coincides with the object.

The surface skeleton of a 3D object is a set of surfaces and curves, symmetrically placed within the object, which has the same topology and reflects the shape of the object. See [8] and the references listed therein for more details on 3D surface skeletonization. If the surface skeleton includes all the CMBs, then the object can be fully reconstructed starting from its surface skeleton. Unit thickness and inclusion of all CMBs are not possible at the same time in presence of object regions whose thickness is given by an even number of voxels. Since the set of the CMBs is at most 2-voxel thick, a nearly-thin skeleton, at most 2-voxel thick, is obtained if full recoverability is desired. A process, generally referred to as final thinning, has to be performed if a unit-thick surface skeleton is requested.

\section{New $3 \times 3 \times 3$ Topology Preserving Removal Operations}

As already pointed out, a voxel p should have a face-neighbor in the background, i.e., $\mathrm{p}$ should be a border voxel, to avoid creation of cavities in $\mathrm{N}(\mathrm{p})$ when $\mathrm{p}$ is removed. Moreover, since we are interested in removal operations to be used in the framework of skeletonization, protrusion voxels cannot be removed. In fact, their presence in the skeleton is necessary to reflect the geometrical properties of the object, e.g., to account for its elongated parts. Thus, to be removable, $\mathrm{p}$ should have an inside faceneighbor.
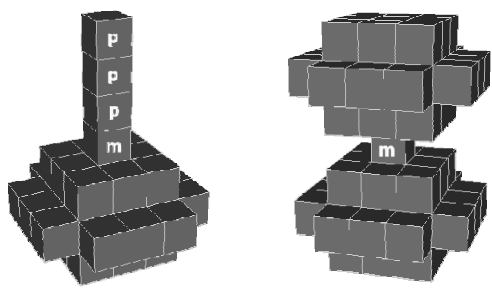

Fig. 1. Voxels denoted by $\mathrm{p}$ form a protrusion, voxels denoted by $\mathrm{m}$ are necessary to maintain connectedness between the protrusion and the inside voxels, left, and between two components of inside voxels, right

To consider $\mathrm{p}$ as a candidate to removal, we request that the above mentioned background and inside face-neighbors of $\mathrm{p}$ form with $\mathrm{p}$ a face-aligned path, i.e. we request that a transition exists for $\mathrm{p}$. It is immediate to see that the existence of a transition for $\mathrm{p}$ guarantees that when $\mathrm{p}$ is removed: 1) it is not an isolated voxel, thus no vanishing of object components occurs, 2) cavities are not created, 3) protrusion voxels are preserved, 4) type 1 tunnels and background fusions are not created in the 
direction of the transition since the two face-neighbors of $\mathrm{p}$ forming a face-aligned path are not both background voxels, and 5) voxels connecting a protrusion and the set of inside voxels, or two components of inside voxels, are preserved, if they are the only voxels ensuring connection, since no transition exists for them. Thus, the remaining conditions to pose for safe removal of $\mathrm{p}$ should guarantee only object connectedness preservation in the remaining directions, and avoid the creation of type 2 tunnels as well as fusion of background components.

For the 3D objects shown in Fig.1, no transition exists for voxels (denoted by p) forming a protrusion and for voxels (denoted by $\mathrm{m}$ ) necessary to maintain connectedness along the face direction between the protrusion and the inside voxels, Fig.1 left, and between two components of inside voxels, Fig. 1 right.

Once the two face-neighbors of $p$ necessary for the existence of a transition have been identified, we describe $\mathrm{N}(\mathrm{p})$ as consisting of three $3 \times 3$ windows. The $3 \times 3$ sets, respectively centered on $\mathrm{p}$, on its background face-neighbor $\mathrm{p}_{\mathrm{T}}$, and on its inside faceneighbor $\mathrm{p}_{\mathrm{B}}$, are called central, top and bottom windows. The remaining elements in the windows are denoted by their cardinal directions with respect to $\mathrm{p}, \mathrm{p}_{\mathrm{T}}$ and $\mathrm{p}_{\mathrm{B}}$, as shown in Fig.2. In this interpretation, independently of the orientation of the actual neighborhood of $\mathrm{p}$, the transition is always seen as occurring from top to bottom.
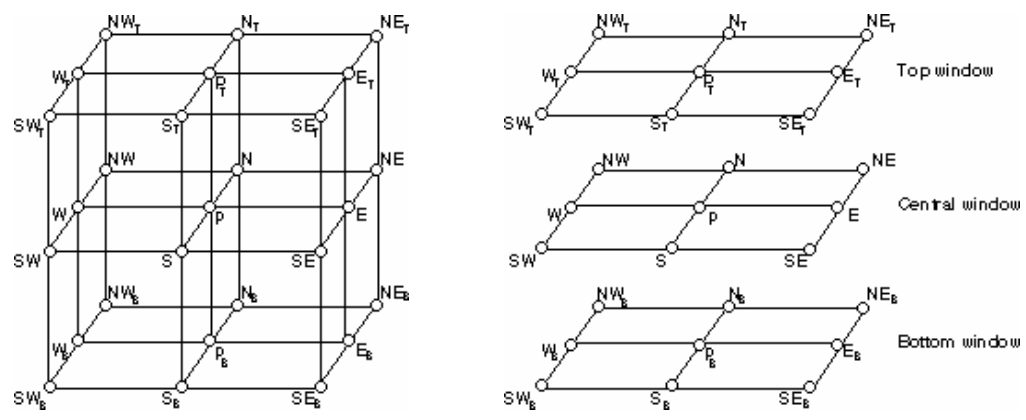

Fig. 2. The $3 \times 3 \times 3$ neighborhood of $\mathrm{p}$ and the top, central and bottom windows constituting it

Since a voxel $\mathrm{p}$ for which a transition exists necessarily has a number $\mathrm{k}=1,2, . ., 5$ of object face-neighbors, we suggest five basic operations for safe removal of $p$, depending on the value of $\mathrm{k}$. Naturally, rotated and mirrored operations have also to be taken into account. The basic removal operations are discussed below with reference to the interpretation of $\mathrm{N}(\mathrm{p})$ into the top, central and bottom windows.

As far as object connectedness preservation is concerned, we note that the object neighbors of $\mathrm{p}$ in the central and bottom windows are all directly 26-connected to the inside voxel $\mathrm{p}_{\mathrm{B}}$. Thus, we should only consider the object neighbors of $\mathrm{p}$ in the top window (of course, we don't consider $\mathrm{p}_{\mathrm{T}}$, which is a background voxel). While vertex-neighbors of $\mathrm{p}$ in the top window play a role only in the object connectedness condition, edge-neighbors of $\mathrm{p}$ in the top window are also important to design a condition preventing background fusion and type 2 tunnel creation, whenever appropriate face-neighbors of $\mathrm{p}$ in the central window are in the background. 


\section{Case k=1}

The only object face-neighbor of $\mathrm{p}$ is $\mathrm{p}_{\mathrm{B}}$ and $\mathrm{p}$ can be removed if $\mathrm{N}_{\mathrm{T}}=0 \wedge \mathrm{E}_{\mathrm{T}}=0 \wedge \mathrm{S}_{\mathrm{T}}=0 \wedge \mathrm{W}_{\mathrm{T}}=0 \wedge\left(\mathrm{NE}_{\mathrm{T}}=0 \vee \mathrm{NE}=1\right) \wedge\left(\mathrm{SE}_{\mathrm{T}}=0 \vee \mathrm{SE}=1\right) \wedge\left(\mathrm{SW}_{\mathrm{T}}=0 \vee \mathrm{SW}=1\right) \wedge$ $\left(\mathrm{NW}_{\mathrm{T}}=0 \vee \mathrm{NW}=1\right)$

Note that $\left(\mathrm{NE}_{\mathrm{T}}=0 \vee \mathrm{NE}=1\right),\left(\mathrm{SE}_{\mathrm{T}}=0 \vee \mathrm{SE}=1\right),\left(\mathrm{SW}_{\mathrm{T}}=0 \vee \mathrm{SW}=1\right)$ and $\left(\mathrm{NW}_{\mathrm{T}}=0 \vee \mathrm{NW}=1\right)$ respectively prevent that object disconnections occur in any of the four possible vertex-directions. Moreover, no background fusion or tunnels are created and no object disconnections occur in the four possible edge-directions, if the edge-neighbors of $\mathrm{p}$ in the top window are in the background, i.e., if $\mathrm{N}_{\mathrm{T}}=0 \wedge \mathrm{E}_{\mathrm{T}}=0 \wedge \mathrm{S}_{\mathrm{T}}=0 \wedge \mathrm{W}_{\mathrm{T}}=0$. In Fig.3, an example of removable voxel is shown for case $\mathrm{k}=1$.

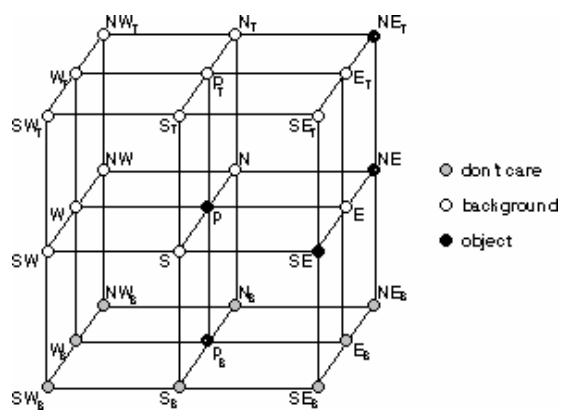

Fig. 3. Case $k=1$. The voxel $p$ with one object face-neighbor, the inside voxel $p_{B}$, is removable. It would not be removable if, still being $\mathrm{NE}_{\mathrm{T}}$ an object voxel, $\mathrm{NE}$ is a background voxel, or if any among $\mathrm{N}_{\mathrm{T}}, \mathrm{E}_{\mathrm{T}}, \mathrm{S}_{\mathrm{T}}$ and $\mathrm{W}_{\mathrm{T}}$ is an object voxel.

\section{Case $\mathrm{k}=2$}

The two object face-neighbors of $\mathrm{p}$, one of which is $\mathrm{p}_{\mathrm{B}}$, cannot form a face-aligned path with $\mathrm{p}$, due to existence of a transition for $\mathrm{p}$. Thus, they are edge-neighbors of each other. Since there exist four possible pairs of edge-connected face-neighbors of $\mathrm{p}$, one of which is $\mathrm{p}_{\mathrm{B}}$, the removal condition is one of the following four conditions, depending on which is the second object face-neighbor of $\mathrm{p}$.

If $\mathrm{N}=1$, the condition is: $\mathrm{W}_{\mathrm{T}}=0 \wedge \mathrm{S}_{\mathrm{T}}=0 \wedge \mathrm{E}_{\mathrm{T}}=0 \wedge\left(\mathrm{SW}=1 \vee \mathrm{SW}_{\mathrm{T}}=0\right) \wedge\left(\mathrm{SE}=1 \vee \mathrm{SE} \mathrm{T}_{\mathrm{T}}=0\right)$

If $\mathrm{W}=1$, the condition is: $\mathrm{N}_{\mathrm{T}}=0 \wedge \mathrm{S}_{\mathrm{T}}=0 \wedge \mathrm{E}_{\mathrm{T}}=0 \wedge\left(\mathrm{SE}=1 \vee \mathrm{SE}_{\mathrm{T}}=0\right) \wedge\left(\mathrm{NE}=1 \vee \mathrm{NE}_{\mathrm{T}}=0\right)$

If $\mathrm{S}=1$, the condition is: $\mathrm{W}_{\mathrm{T}}=0 \wedge \mathrm{N}_{\mathrm{T}}=0 \wedge \mathrm{E}_{\mathrm{T}}=0 \wedge\left(\mathrm{NW}=1 \vee \mathrm{NW}_{\mathrm{T}}=0\right) \wedge\left(\mathrm{NE}=1 \vee \mathrm{NE}_{\mathrm{T}}=0\right)$

If $\mathrm{E}=1$, the condition is: $\mathrm{W}_{\mathrm{T}}=0 \wedge \mathrm{S}_{\mathrm{T}}=0 \wedge \mathrm{N}_{\mathrm{T}}=0 \wedge\left(\mathrm{NW}=1 \vee \mathrm{NW}_{\mathrm{T}}=0\right) \wedge\left(\mathrm{SW}=1 \vee \mathrm{SW}_{\mathrm{T}}=0\right)$

\section{Case $\mathrm{k}=3$}

Due to the transition from $\mathrm{p}_{\mathrm{T}}$ to $\mathrm{p}_{\mathrm{B}}$, the remaining two object face-neighbors of $\mathrm{p}$ are necessarily placed in the central window and either are edge-neighbors of each other (four possible configurations), or form a face-aligned path with $\mathrm{p}$ (two possible configurations). Thus, the removal condition is one of the following six conditions, depending on which are the two object face-neighbors of $\mathrm{p}$ in the central window.

If $\mathrm{W}=1$ and $\mathrm{S}=1$, the condition is: $\mathrm{N}_{\mathrm{T}}=0 \wedge \mathrm{E}_{\mathrm{T}}=0 \wedge\left(\mathrm{NE}_{\mathrm{T}}=0 \vee N E=1\right)$

If $\mathrm{W}=1$ and $\mathrm{N}=1$, the condition is: $\mathrm{S}_{\mathrm{T}}=0 \wedge \mathrm{E}_{\mathrm{T}}=0 \wedge\left(\mathrm{SE}_{\mathrm{T}}=0 \vee \mathrm{SE}=1\right)$

If $\mathrm{N}=1$ and $\mathrm{E}=1$, the condition is: $\mathrm{S}_{\mathrm{T}}=0 \wedge \mathrm{W}_{\mathrm{T}}=0 \wedge\left(\mathrm{SW}_{\mathrm{T}}=0 \vee \mathrm{SW}=1\right)$ 
If $\mathrm{E}=1$ and $\mathrm{S}=1$, the condition is: $\mathrm{N}_{\mathrm{T}}=0 \wedge \mathrm{W}_{\mathrm{T}}=0 \wedge\left(\mathrm{NW}_{\mathrm{T}}=0 \vee \mathrm{NW}=1\right)$

If $\mathrm{W}=1$ and $\mathrm{E}=1$, the condition is: $\mathrm{N}_{\mathrm{T}}=0 \wedge \mathrm{S}_{\mathrm{T}}=0$

If $\mathrm{N}=1$ and $\mathrm{S}=1$, the condition is: $\mathrm{E}_{\mathrm{T}}=0 \wedge \mathrm{W}_{\mathrm{T}}=0$

Case $\mathrm{k}=4$

Except for $p_{B}$, which is in the bottom window, the remaining three object faceneighbors of $\mathrm{p}$ are all in the central window (four possible configurations). Thus, the removal condition is one of the following four conditions, depending on the positions of the three object face-neighbors of $\mathrm{p}$ in the central window.

If $\mathrm{W}=1, \mathrm{E}=1$, and $\mathrm{S}=1$, the condition is: $\mathrm{N}_{\mathrm{T}}=0$

If $\mathrm{N}=1, \mathrm{E}=1$, and $\mathrm{S}=1$, the condition is: $\mathrm{W}_{\mathrm{T}}=0$

If $\mathrm{W}=1, \mathrm{E}=1$, and $\mathrm{N}=1$, the condition is: $\mathrm{S}_{\mathrm{T}}=0$

If $\mathrm{W}=1, \mathrm{~N}=1$, and $\mathrm{S}=1$, the condition is: $\mathrm{E}_{\mathrm{T}}=0$

\section{Case $\mathrm{k}=5$}

Except for $\mathrm{p}_{\mathrm{B}}$, which is in the bottom window, the remaining four object faceneighbors of $\mathrm{p}$ are all in the central window and $\mathrm{p}$ is always removable.

In Cases $k=2, k=3$ and $k=4$, more than one transition is possible. In these cases, the relative above condition should be checked in correspondence with any existing transition. It is straightforward to see that if the proper condition is satisfied in correspondence with one transition, $\mathrm{p}$ can be safely removed.

In the following we will denote by $f$ the removal operator that includes detection of transitions and involves the five cases $k=1-5$. A voxel $p$ is removed if the operator finds out that at least a transition exists for $\mathrm{p}$ and the proper removal condition, based on the number of object face-neighbors of $\mathrm{p}$, is satisfied. We remind that voxels constituting object protrusions are not removed by $f$ since no transition exist for them. This is an important feature to use $f$ in skeletonization, since it guarantees that no ad hoc criterion is necessary to prevent unwanted shortening of peripheral surfaces/branches of the skeleton.

For simplicity, we will refer to $\left(\mathrm{NE}_{\mathrm{T}}=0 \vee \mathrm{NE}=1\right),\left(\mathrm{SE}_{\mathrm{T}}=0 \vee \mathrm{SE}=1\right),\left(\mathrm{SW}_{\mathrm{T}}=0 \vee \mathrm{SW}=1\right)$ and $\left(\mathrm{NW}_{\mathrm{T}}=0 \vee \mathrm{NW}=1\right)$ as to vertex conditions and to the conditions $\mathrm{N}_{\mathrm{T}}=0, \mathrm{E}_{\mathrm{T}}=0, \mathrm{~S}_{\mathrm{T}}=0$, and $\mathrm{W}_{\mathrm{T}}=0$ as to edge conditions. Note that the argument involved in an edge condition is the only one relative to the edge-neighbor in the top window of p such that the corresponding voxel vertically aligned with it, i.e., the face-neighbor of $\mathrm{p}$ in the central window, is a background voxel.

\section{Surface Skeletonization by Sequential Voxel Removal}

In surface skeletonization by sequential voxel removal, each currently inspected border voxel is removed, i.e., is set to 0 , if the proper condition is satisfied. Of course, to guarantee that the surface skeleton is centrally placed within the object, an iterative process is necessary to candidate to removal at each iteration only voxels belonging to the current border of the object. To save computation time and have all borders directly available, the distance transform DT is used. The border of the object at the $l$-th iteration includes all voxels with distance label $l$ in DT, as well as voxels with 
distance label smaller than $l$, if these latter voxels were not removed during previous iterations. Voxels with label greater than $l$ are inside voxels at the $l$-th iteration. The background is, at all iterations, the current set of 0's. A transition exists for a voxel p at the $l$-th iteration, if $\mathrm{p}$ has two face-neighbors forming with $\mathrm{p}$ a face-aligned path such that one of these neighbors is 0 and the other has label $l+1$.

The algorithm can be sketched as follows.

Compute DT. Let $m$ be the maximal distance label in DT for $l=1$ to $m$ do apply $f$ to any voxel $\mathrm{p}$ with distance label $l$, and set $\mathrm{p}$ to 0 , if removable.

It is easy to see that CMBs are voxels for which no transition occurs. In fact, none of the face-neighbors of a CMB can be an inside voxel. Thus, the obtained surface skeleton SK includes all the CMBs and complete recovery of the object is possible. SK is likely to be 2-voxel thick in some parts and final thinning is necessary if unit thickness is a desired feature.

An example of the performance of the algorithm is given in Fig.4.
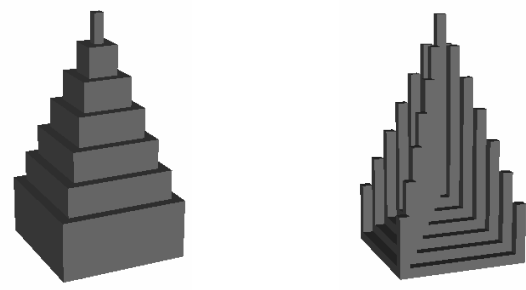

Fig. 4. Object, left, and its surface skeleton, right

We point out that small asymmetries could exist in the resulting SK. These asymmetries occur only in presence of pairs of face-connected border voxels such that only one of them is removable, depending on the order in which voxels are inspected. See Fig.5. There, the central section of a small 3D object is shown; gray voxels are inside voxels, all other voxels belong to the border. The two sections before and after the central section include only two voxels each; these voxels are the face-neighbors of the voxels shown in gray in the central section. Depending on the order in which voxels are examined, removal of the voxel $k(h)$ will allow a transition for the voxel $p(q)$. The voxel p (q) is thus removed since the proper removal condition is satisfied.

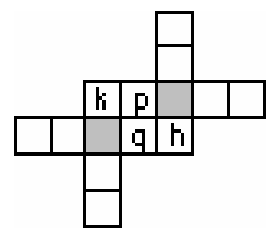

Fig. 5. Section of a 3D object, where inside voxels are gray. Voxels k, p and h (q, h and k) are removed if the section is examined in forward (backward) fashion. 
To avoid creation of asymmetries, one could detect parallelwise, at each iteration, the voxels for which a transition exists. For the example in Fig.5, both $\mathrm{p}$ and $\mathrm{q}$ would remain in the skeleton. In fact, $\mathrm{p}$ and $\mathrm{q}$ belong to the same current border including $\mathrm{k}$ and $\mathrm{h}$, and no transition would exist for them. We point out that a parallel detection of voxels for which a transition exists implies doubling the number of iterations necessary to obtain SK. Moreover, other asymmetries are likely to be created by final thinning when a unit-thick skeleton is desired. Thus, especially in this latter case, resorting to parallel detection of transitions is not convenient.

For the sake of completeness we point out that, due to the implicit selection of all CMBs as skeletal voxels, the surface skeleton may include unwanted peripheral branches/surfaces. These are originated in the presence of noisy border configurations in correspondence of which some CMBs, generally characterized by small distance values, are found. Thus, as it is always the case for skeletonization, some pruning should be applied to remove noisy peripheral branches/surfaces (see e.g., [9]).

\section{Surface Skeletonization by Parallel Voxel Removal}

In Section 3, we have introduced the removal operator $f$ that does not alter topology when sequentially applied. We discuss here the behavior of $f$, when applied in parallel in the framework of iterative skeletonization.

An immediate remark is that a fully parallel skeletonization algorithm, i.e., an algorithm based on the use of just one completely parallel operator with $3 \times 3 \times 3$ support [10], cannot be designed. In fact, the application of the operator $f$ requires that object voxels be distinguished in border voxels and inside voxels, since the existence of a transition must be checked. Thus, each iteration of the skeletonization algorithm must include a sub-iteration devoted to the identification of the border voxels.

It is straightforward to observe that object vanishing and creation of cavities cannot occur, since no transition exists for voxels of object parts entirely consisting of border voxels and for inside voxels. A formal proof that $f$ does not alter object connectedness and does not create fusion of background components, when two neighboring border voxels $\mathrm{p}$ and $\mathrm{q}$ are simultaneously removed, can be found in [11]. In fact, proving that $f$ does not alter object and background connectedness in the set $N(p) \cup N(q)$ is enough to guarantee that object and background connectedness are also globally preserved. Here we just point out that, although our removal operations apparently have only a $3 \times 3 \times 3$ support, the existence of the transition for a voxel $\mathrm{p}$ candidate to removal implies that at least a face-neighbor of $\mathrm{p}$ is an inside voxel and, hence, the operations implicitly use a support larger than $3 \times 3 \times 3$. In [11], the worst case, occurring when a candidate voxel has only one inside face-neighbor, is discussed.

As regards the possibility to create tunnels by simultaneous removal of two neighboring object voxels $\mathrm{p}$ and $\mathrm{q}$, we note that $\mathrm{p}$ and $\mathrm{q}$ must obviously be faceneighbors of each other. Indeed, it can be seen by examining Fig.6 that tunnels, not corresponding to real tunnels in the original object, can be created under some circumstances by simultaneous removal of two face-adjacent voxels $\mathrm{p}$ and $\mathrm{q}$.

A suitable operator $g$, to detect the configurations that could create tunnels has to be designed. Such an operator can be employed only after the operator $f$ has partitioned the set of border voxels into removable and non-removable voxels, since 
spurious tunnels can be eliminated only after the configurations that would cause them have been created. Thus, the identification on the current border of which voxels to remove should be done in two sub-iterations. During the first sub-iteration $f$ is applied to mark non-removable border voxels as belonging to the skeleton. Obviously, border voxels that have already been assigned to the skeleton at some previous iteration do not need to be newly checked by $f$. During the second sub-iteration, the operator $g$ is applied to the non-marked border voxels to mark as non-removable (and, hence, as belonging to the skeleton) all the voxels whose removal would create tunnels.
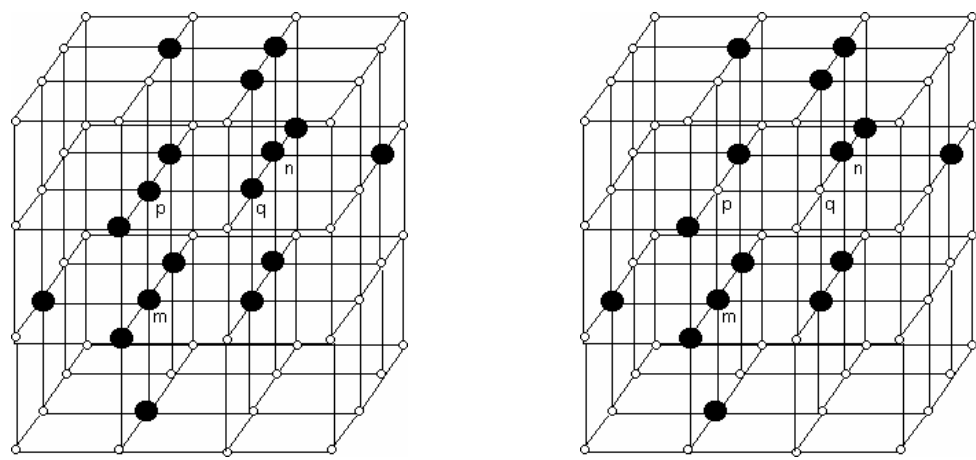

Fig. 6. The 3D configuration, where black dots are object voxels and $\mathrm{m}$ and $\mathrm{n}$ are inside voxels, before (left) and after (right) simultaneous removal of $\mathrm{p}$ and $\mathrm{q}$

We have examined the various configurations embedding a pair of face-adjacent voxels $\mathrm{p}$ and $\mathrm{q}$ to identify the cases in which the operator $f$ fails to preserve topology when applied in parallel, so as to find the adequate operator $g$. Actually, in [11] we show that only one basic configuration exists, where the operator $f$ fails.

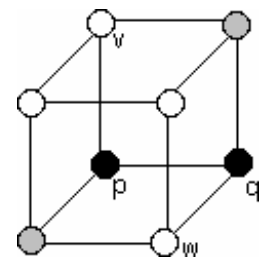

Fig. 7. Basic configuration where parallel removal of $\mathrm{p}$ and $\mathrm{q}$ causes spurious tunnel creation. Gray voxels are voxels already assigned to the skeleton

The basic configuration is shown in Fig.7, where the two voxels $v$ and w are background voxels (by the way, they are necessary for the transitions relative to $\mathrm{p}$ and $\mathrm{q}$ ), the two voxels which are face-neighbors of both $\mathrm{p}$ and $\mathrm{w}$, and of both $\mathrm{q}$ and $\mathrm{v}$, respectively (shown in gray) have already been assigned to the skeleton (during any previous iteration, or during the sub-iteration of the current iteration in which $f$ has been 
applied) and the remaining two voxels (denoted for simplicity by white dots like $\mathrm{v}$ and w) are don't care. Since it is enough that one of the two voxels p and q is marked as non-removable to avoid creation of a spurious tunnel, the number of mirrored and rotated configurations figures up at 24 . The $3 \times 3 \times 3$ operator $g$ is designed by taking into account the basic configuration shown in Fig.7, as well as the configurations derived from it.

For example, with reference to Fig.2, if $\mathrm{p}$ has $\mathrm{q}$ as its face-neighbor $\mathrm{E}$ in the central window, the neighbors of $\mathrm{p}$ that are actually checked by the operator $g$ in $\mathrm{N}(\mathrm{p})$ are $\mathrm{q}$, $\mathrm{p}_{\mathrm{T}}, \mathrm{E}_{\mathrm{T}}, \mathrm{S}$, and SE. The voxel $\mathrm{p}$ is marked as non-removable if $\mathrm{q}$ is a non-marked border voxel, $\mathrm{p}_{\mathrm{T}}$ and $\mathrm{SE}$ are background voxels, and $\mathrm{E}_{\mathrm{T}}$ and $\mathrm{S}$ are marked voxels.

In summary, each iteration of the skeletonization algorithm can be outlined as follows.

Distinguish the object voxels into border, skeletal and inside voxels.

Apply $f$ to the border voxels and, for any voxel identified as non-removable, change its status from border voxel to skeletal voxel.

Apply $g$ to the border voxels and, for any voxel identified as non-removable, change its status from border voxel to skeletal voxel.

Remove all border voxels.

Skeletonization terminates when no voxels are removed, i.e., all voxels in the current border have been changed into skeletal voxels.

An example of the performance of the parallel algorithm is given in Fig.8.
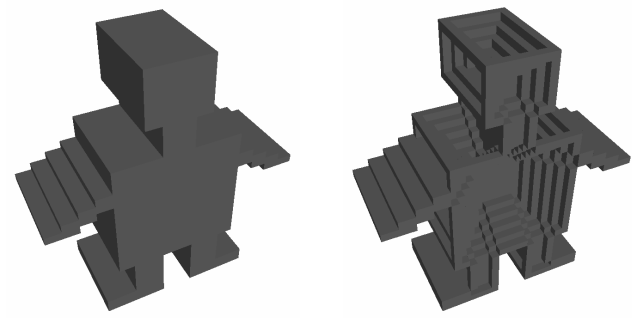

Fig. 8. Object, left, and its surface skeleton, right

In particular, when implementing the parallel algorithm on a standard sequential computer by scanning the 3D array in the top-bottom, left-right and front-back directions, as soon as the operator $g$ identifies a voxel $p$, whose neighborhood satisfies any of the 24 configurations, $\mathrm{p}$ can be marked together with the relative face-neighbor $\mathrm{q}$. In this way, both voxels in the pair matching the configurations derived from Fig.7 are marked, and a perfectly symmetrical surface skeleton is obtained. Moreover, DT can be conveniently used to reduce the computational effort. In fact, instead of including at each iteration the sub-iteration necessary to distinguish border voxels and inside voxels (which would require one scan of the image), distinction between border and inside voxels is available at all iterations once DT is computed (in two scans of the image). At the $l$-th iteration, the border includes all voxels with distance label $l$ in DT 
as well as all voxels with distance label smaller than $l$ and assigned to the skeleton during some previous iteration. The surface skeleton SK results to be centered within the object, includes all CMBs, is at most 2-voxel thick and is completely symmetrical.

\section{Final Thinning}

The surface skeleton obtained by using sequential or parallel voxel removal is expected to consist only of border voxels. However, inside voxels can still be present in SK. For example, this is the case for the object (coinciding with its surface skeleton) shown in Fig.9, which consists of three bricks, each with section $2 \times 2$ voxels and mutually crossing each other, where the kernel of the crossing region includes eight inside voxels. Another example is given by complex objects including irreducible sets, i.e., objects such that the only way to obtain a unit-thick SK would be that of forcing topology changes. We do not consider the case of irreducible sets here (for a discussion of this case in 2D see [12]) and refer to the standard case, where a few inside voxels exist only in the presence of crossings.

Both the algorithm based on sequential removal and the one based on parallel removal require final thinning if unit thickness is desired for SK. We describe below a final thinning algorithm based on sequential removal.

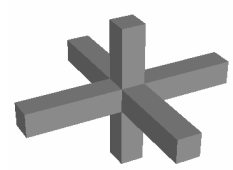

Fig. 9. A nearly-thin surface skeleton including eight inside voxels

Since the only voxels that should be removed by this process are those located where the object is 2-voxel thick in face-direction, we should detect the existence of such subsets of SK. To this purpose, we use six $4 \times 1$ masks, each consisting of four voxels forming a face-aligned path along one of the three principal directions (topbottom or bottom-top, left-right or right-left, and front-back or back-front). In each mask, the two external voxels are background voxels and the two internal ones, say $v_{1}$ and $\mathrm{v}_{2}$, are object voxels.

A simplified version $f^{\prime}$ of the operator $f$ is used to perform final thinning along the direction where 2-voxel thickness is identified by one of the above masks. Of course, no transition exists in the direction of the mask, since SK is nearly-thin in that direction, but the voxel $\mathrm{v}_{2}\left(\mathrm{v}_{1}\right)$ plays the role of inside voxel in $\mathrm{N}\left(\mathrm{v}_{1}\right)\left(\mathrm{N}\left(\mathrm{v}_{2}\right)\right)$. The operator $f$ ' removes $\mathrm{v}_{1}\left(\mathrm{v}_{2}\right)$, depending on the number of object face-neighbors in $\mathrm{N}\left(\mathrm{v}_{1}\right)$ $\left(\mathrm{N}\left(\mathrm{v}_{2}\right)\right)$.

Final thinning is done in two scans. During the first scan, the three masks detecting 2-voxel thickness along the top-bottom, left-right and front-back directions are used. During the second scan, the remaining three masks are used.

If, during the first scan, for the current voxel $v_{1}$ the mask is matched, but $v_{1}$ is not removed by the operator $f$, the coordinates of $\mathrm{v}_{2}$ are recorded to directly access that 
voxel during the second scan. In this way, the mask does not need to be newly checked and the second scan is avoided.

Final thinning should be applied twice since, as said above, the nearly-thin skeleton might include some inside voxels, which could become removable border voxel, after removal of some of their neighbors.

\section{Conclusion}

We have introduced two $3 \times 3 \times 3$ operators $f$ and $g$. Only $f$ is necessary if skeletonization is accomplished by sequential voxel removal. Both operators are used to design an iterative parallel algorithm for surface skeletonization. The obtained surface skeleton has all the expected properties except for unit thickness, as it is 2-voxel thick wherever the thickness of the object is expressed by an even number of voxels. In this respect, we have also introduced a sequential final thinning algorithm, which originates the unit-thick surface skeleton.

\section{References}

1. Rosenfeld, A.: A characterization of parallel thinning algorithms. Information and Control 29 (1975) 286-291

2. Tsao, Y.F., Fu, K.S.: A parallel thinning algorithm for 3-D pictures. Computer Graphics and Image Processing 17 (1981) 315-331

3. Saha, P.K., Chaudhuri, B.B.: Detection of 3D simple points for topology preserving transformations with application to thinning. IEEE Trans. PAMI 16 (1994) 1028-1032

4. Bertrand, G., Malandain, G.: A new characterization of three-dimensional simple points. Pattern Recognition Letters 15 (1994) 169-175

5. Kong, T.Y.: A digital fundamental group. Computers and Graphics. 13 (1989) 159-166

6. Kong, T.Y., Rosenfeld, A.: Appendix: Digital Topology - A brief introduction and bibliography. In: Kong, T.Y., Rosenfeld, A (eds.): Topological Algorithms for digital image processing. Elsevier, Amsterdam (1996) 263-292

7. Borgefors, G.: On digital distance transform in three dimensions. Computer Vision and Image Understanding. 64(3) (1996) 368-376

8. Sanniti di Baja, G., Nystrom, I.: Skeletonization in 3D discrete binary images. In Chen, C.H., Wang, P.S.P. (eds.): Handbook of Pattern Recognition and Computer Vision. World Scientific, Singapore (2005) 137-156

9. Borgefors, G., Nyström, I., Svensson, S., Sanniti di Baja, G.: Simplification of 3D skeletons using distance information. In Latecki, L.J. et al. (eds.): Vision Geometry IX, Proc. SPIE 4117 (2000) 300-309

10. Hall, R.W.: Parallel connectivity-preserving thinning algorithms. In: Kong, T.Y., Rosenfeld, A (eds.): Topological Algorithms for digital image processing. Elsevier, Amsterdam (1996) 145-179

11. Arcelli, C., Sanniti di Baja, G., Serino, L.: Topology preservation in 3D” Internal Report R.I.161/05, Istituto di Cibernetica, December 2005

12. Eckhardt, U., Latecki, L., Maderlechner, G.: Irreducible and thin binary sets. In Arcelli, C. et al. (eds.): Aspects of Visual Form Processing. World Scientific, Singapore (1994) 199-208 